\title{
Imazamethabenz inhibits human breast cancer cell proliferation, migration and invasion via combination with Pin1
}

\author{
CHEN LIU, CHAOYU MU, ZENG LI and LIANG XU \\ College of Pharmacy, Anhui Medical University, Hefei, Anhui 230032, P.R. China \\ Received February 11, 2016; Accepted February 9, 2017
}

DOI: $10.3892 / \mathrm{mmr} .2017 .6399$

\begin{abstract}
Overexpression of peptidyl-prolyl cis/trans isomerase, NIMA interacting-1 (Pin1) is a significant marker of the occurrence and development of tumors. In the present study, the imidazoline ketone herbicide imazamethabenz was investigated as a potential antitumor drug by inhibiting Pin1. Molecular docking and enzyme activity tests verified, for the first time, that the imidazoline ketone compound imazamethabenz effectively inhibited Pin1 in vitro. MTT assays, western blotting, wound healing assay and Matrigel invasion assays revealed that imazamethabenz induced apoptosis and inhibited migration and invasion of the breast cancer cell line MCF-7, which overexpresses Pin1, by inhibiting the Pin1-mediated signaling pathway involving vascular endothelial growth factor and matrix metalloproteinase 9. These findings indicated that imazamethabenz offers potential applications for the treatment of tumors as a Pin1 inhibitor.
\end{abstract}

\section{Introduction}

Peptidyl-prolyl cis/trans isomerase, NIMA interacting-1 (Pin1), a peptide-prolyl amide linkage isomerase discovered in 1996, is a protein that regulates mitosis. Pin 1 also regulates the conformation of phosphorylatable substrates and influences their physiological function by specifically catalyzing the cis-trans isomerism of the phosphorylated (p)Ser/Thr-Pro amide linkage (1). Previous biological research has indicated that Pin 1 is overexpressed in multiple tumor cells, including cervical cancer, prostate cancer, lung cancer, breast cancer, liver cancer and melanoma (2-5). Overexpression of Pin1 is a significant marker of the occurrence and development of tumors (2-5). Multiple known oncogenes and cancer suppressor proteins are substrates of Pin1. Pin1 activates the protein activity of oncogenes and promotes the occurrence

Correspondence to: Dr Zeng Li, College of Pharmacy, Anhui Medical University, 81 Meishan Road, Hefei, Anhui 230032, P.R. China

E-mail: lizeng@ahmu.edu.cn

Key words: imazamethabenz, peptidyl-prolyl cis/trans isomerase, VEGF, MMP9, antitumor and development of tumors by regulating the conformation of phosphorylatable substrates (6-7). Hence, Pin1 inhibition is a possible novel mechanism to exploit for antitumor therapy.

Research on crystal structures of a known Pin1 small-molecule inhibitor and Pin 1 compounds has demonstrated that the pharmacophore characteristics of this inhibitor are two hydrophobic centers and one H-bond acceptor (8-10). Pin1 binds to the $\mathrm{H}$-bond acceptor in the small-molecule inhibitor by binding the side chains of basic amino acid residues, namely, Lys63, Arg68, and Arg6, to the cavity. Thus, Pin1 contributes to the orientation and binding of small molecules in the binding cavity. His59, His157, Met130 and Phe134 constitute a hydrophobic center, which is usually defined as the prolyl pocket. The other hydrophobic center is a flat region that consists of His59, Ala118 and Leu122; this center binds to bulk-mass aromatic structural segments. By investigating and screening this structure, the imidazoline ketone herbicide, imazamethabenz was demonstrated to exhibit the capacity to bind to Pin1 (11). The present study demonstrated that this compound inhibits the activity of Pin1 in vitro. MTT cytotoxicity tests, caspase-3 enzyme expression quantity tests and cellular migration and invasion tests verified that imazamethabenz effectively inhibited the proliferation, migration and invasion of the human breast cancer cell line MCF-7. This result provides a basis for developing novel types of Pin1 inhibitors.

\section{Materials and methods}

Materials. Imazamethabenz and 3-(4,5-Dimethylthiazol-2-yl)2,5-diphenyltetrazolium bromide (MTT) were purchased from Aladdin Shanghai Biochemical Technology Co., Ltd. (Shanghai, China). Stock solutions of compounds (10 mM) were prepared in DMSO and stored at $-80^{\circ} \mathrm{C}$. The solution was diluted to working concentrations using double-distilled, deionized water. Pin 1 in prokaryotic expression vector pMAL-c2X and the expression in $E$. coli and purification of the Pin1 fusion protein as previously been described by Zhang et al (12). The synthetic substrate peptide [suc-Ala-Glu-Pro-Phe-peptide nucleic acid (pNA)] and bovine serum albumin were purchased from Sangon Biotech Co., Ltd. (Shanghai, China). The MCF-7 cell line was obtained from the Animal Experimental Center of Sun Yat-Sen University (Guangzhou, China). All antibodies were purchased from Sigma-Aldrich; Merck KGaA (Darmstadt, Germany). 
Molecular docking. The X-ray crystal structure of Pin1 complexed with the carboxylate inhibitor was obtained from the RCSB Protein Data Bank (PDB code: 3JYJ) (13). The docking studies were predicted using AUTODOCK 4.0 software (The Scripps Research Institute, La Jolla, CA, USA). The dimensions of the active site box were selected to be large enough to contain the entire protein molecule. All docking calculations were performed using a Lamarckian genetic algorithm. The default FlexX parameters were used. The most likely conformation of the ligand was selected based on its binding affinity and optimal binding arrangement with the hydrophobic centers and H-bond acceptors of Pin1.

Inhibitory activity of Pinl enzyme. Pin1 inhibitory activity was evaluated through the proteinase-coupled method (14). A mixture of $250 \mu \mathrm{M}$ synthetic substrate peptide [suc-Ala-Glu-Pro-Phe-peptide nucleic acid (pNA)], $0.2 \mathrm{mM}$ dithiothreitol, $100 \mu \mathrm{g} / \mathrm{ml}$ bovine serum albumin, and $25 \mathrm{nM}$ Pin1 in $150 \mu 135 \mathrm{mM}$ HEPES-KOH (pH 7.8) was added to the DMSO solution. The indicated concentrations $(0,0.5,2.5$, $5,10,20,4080 \mu \mathrm{M})$ of imazamethabenz were then added to the solution. The mixture was preincubated for $10 \mathrm{~min}$ at $10^{\circ} \mathrm{C}$. The hydrolysis of the substrate peptide was initiated by adding an excess amount of $\alpha$-chymotrypsin $(150 \mu \mathrm{l})$ of $0.8 \mathrm{mg} / \mathrm{ml}$ protease in $35 \mathrm{mM}$ HEPES-KOH (pH 7.8). The absorbance of the released p-nitroaniline at $390 \mathrm{~nm}$ was determined every second for 10 min with an Agilent 8453 UV-vis spectrophotometer (Agilent Technologies, Inc., Santa Clara, CA, USA). Initially, $\alpha$-chymotrypsin rapidly digested the substrate peptides in the trans form, then subsequently hydrolyzed the peptides as they were isomerized from the cis form to the trans form by Pin1. The observed reaction rate of the isomerization phase was designated as Pin1 activity. Inhibitory activity was calculated and expressed as $\left(k_{(\mathrm{inh})}-k_{0}\right) /\left(k_{(\text {noinh })}-k_{0}\right) \times 100(\%)$; where $k_{(\mathrm{inh})}$ is the observed pseudo-first-order rate constant in the presence of the inhibitor, $k_{\text {(noinh) }}$ is the rate constant without the inhibitor and $k_{0}$ is the constant in the absence of Pin1.

MTT assay. MCF-7 cells were seeded in 96-well plates at a concentration of $5 \times 10^{3}$ cells/well and exposed to various concentrations of imazamethabenz $(0.1,0.5,2.5,5,10,20,40$ and $80 \mu \mathrm{M})$. Following treatment for $48 \mathrm{~h}, 10 \mu \mathrm{l}$ MTT solution was added to each well and then incubated for $4 \mathrm{~h}$. Following this, $100 \mu 1$ DMSO was added to each well. Optical density was detected at $490 \mathrm{~nm}$. Three independent experiments were performed. $\mathrm{IC}_{50}$ values were calculated using Origin 8.0 software (OriginLab Corporation, Northampton, MA, USA).

Western blot. Following $4 \mathrm{~d}$ of treatment with or without imazamethabenz $(0,0.5,1$ and $2.5 \mu \mathrm{M})$, the MCF-7 cells were harvested and washed with PBS (pH 7.4) three times. The collected cells were lysed in $150 \mu \mathrm{l}$ of extraction buffer consisting of $100 \mu \mathrm{l}$ solution A (50 mM glucose, $25 \mathrm{mM}$ Tris- $\mathrm{HCl}, \mathrm{pH} 8,0 \mathrm{mM}$ EDTA and $1 \mathrm{mM}$ phenylmethylsulfonyl fluoride) and $50 \mu \mathrm{l}$ solution $\mathrm{B}(50 \mathrm{mM}$ Tris- $\mathrm{HCl}, \mathrm{pH} 6.8,6 \mathrm{M}$ urea, 6\% 2-mercaptoethanol, 3\% sodium dodecyl sulfate and $0.003 \%$ bromophenol blue). The soliquid was then centrifuged at $11,310 \times \mathrm{g}$ at $4^{\circ} \mathrm{C}$ for $5 \mathrm{~min}$. The supernatant (10 $\mu$ l for each sample) was loaded onto $10 \%$ polyacrylamide gel and transferred to a microporous polyvinylidene difluoride membrane. After blocking non-specific binding with 5\% BSA (room temperature for $1 \mathrm{~h}$ ), the membranes were subsequently incubated with primary antibodies against vascular endothelial growth factor (VEGF; cat. no. V4758; 1:500; Sigma-Aldrich, Merck KGaA), matrix metalloproteinase 9 (MMP-9; cat. no. SAB4501896; 1:500; Sigma-Aldrich, Merck KGaA) and $\beta$-actin (cat. no. A1978; 1:1,000; Sigma-Aldrich, Merck KGaA), overnight at $4^{\circ} \mathrm{C}$, followed by incubation with horseradish peroxidase-conjugated anti-mouse (cat. no. A9044; 1:1,000; Sigma-Aldrich, Merck KGaA) or anti-rabbit secondary antibody (cat. no. A4914; 1:1,000; Sigma-Aldrich, Merck $\mathrm{KGaA}$ ) at room temperature for $30 \mathrm{~min}$. The signals were detected using an enhanced chemiluminescence substrate (Bioss Biotechnology, Beijing, China).

Wound healing assay. This assay was performed as previously described (15). The cells were grown to $100 \%$ confluence in 12 -well plates for $24 \mathrm{~h}$. The cell monolayer was scratched with a $200 \mu \mathrm{l}$ pipette tip to obtain constant widths. The cells were then incubated with 0,1 and $2.5 \mu \mathrm{l}$ of imazamethabenz for $1 \mathrm{~h}$ at $37^{\circ} \mathrm{C}$. Cell migration was visualized at magnification x 200 and photographed after $24 \mathrm{~h}$ using a Nikon Eclipse $80 \mathrm{i}$ light microscope (Nikon Corporation, Tokyo, Japan). The wound area was quantified using the program ImageJ version 2.0 (NIH, Bethesda, MD, USA).

Matrigel invasion assay. This assay was performed as previously described (15). Matrigel invasion assays were performed using a 12-well Transwell insert with $8 \mu \mathrm{m}$ pore size from Guangzhou RiboBio Co., Ltd. (Guangzhou, China). The upper wells of the inserts were coated with $100 \mu 1$ Matrigel $(1 \mathrm{mg} / \mathrm{ml})$ and incubated at $37^{\circ} \mathrm{C}$ for $4 \mathrm{~h}$. The coating was then washed once with serum-free medium. MCF-7 cells $\left(1 \times 10^{5}\right.$ cells) with or without imazamethabenz $(0,1$ and $2.5 \mu \mathrm{M})$ and $0.2 \mathrm{ml}$ Dulbecco's modified Eagle's medium (Meilun Biology Technology, Dalian, China) were added to the well. Following incubation for $48 \mathrm{~h}$, the cells in the top well were removed. The cells on the underside of the membrane were dyed using $0.4 \mathrm{~g} / \mathrm{l}$ crystal violet (Aladdin Shanghai Biochemical Technology Co., Ltd.) and counted by light microscopy based on 5 randomly selected fields at magnification $x 200$. Three independent experiments were performed.

Statistical analysis. Each experiment was performed 3-4 times. All data were analyzed by the SPSS 17.0 software (SPSS, Inc., Chicago, IL, USA). The statistical significance of differences was determined by Student's t-test for comparison between one-way analysis of variance. $\mathrm{P}<0.05$ was considered to indicate a statistically significant difference.

\section{Results and Discussion}

Molecular docking. The present study adopted the AUTODOCK 4.0 docking program and RCSB Protein Data Bank (PDB code: 3JYJ) (13), and investigated the effect of binding between imazamethabenz and Pin1, providing a basis for structural modification.

Docking results revealed that imazamethabenz and Pin1 exhibited a good binding effect (Fig. 1). Hydroxyl in the oxalate base and Pin1 bound to the characteristic basic aminoacid 

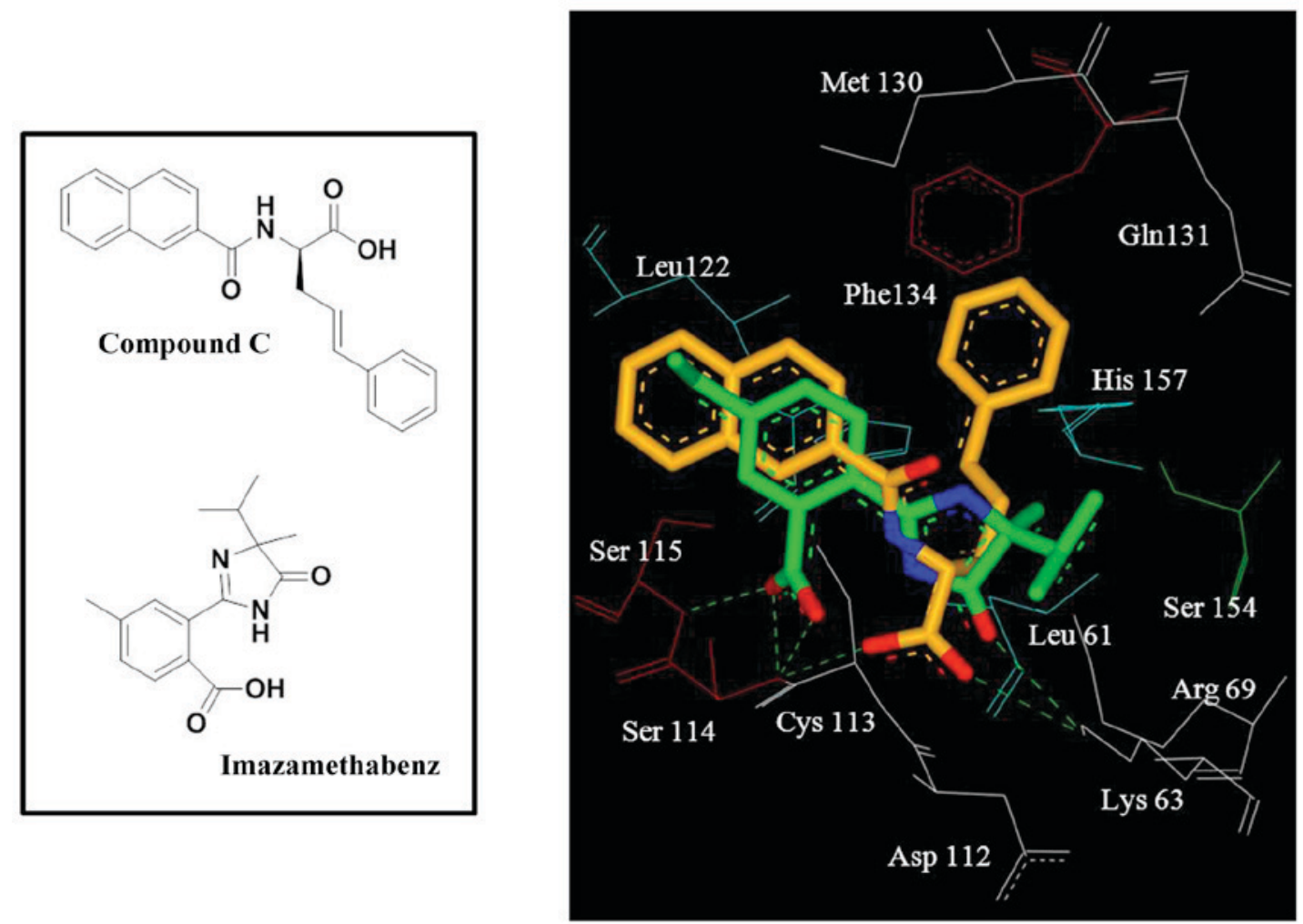

Figure 1. Chemical structures and FlexX-modeled binding modes of the compound imazamethabenz (carbon atoms in green) compared with the crystal structure (PDB code: $3 \mathrm{JYJ}$ ) of compound $\mathrm{C}$ (carbon atoms in orange). H-bonding interactions are presented with green lines.

residue Ser114 to form hydrogen-bond interactions in the cavity and 2-carbonyl in the oxalate base. Furthermore, Ser114 and Ser115 formed a hydrogen bond, and carbonyl in imidazoline ketone and Lys63 formed a hydrogen bond. These findings indicated that the oxalate base is a hydrogen-bond acceptor of the Pin1 inhibitor. The benzene ring reached out to a relatively flat hydrophobic region, which consisted of side chains Leu122 and Cys113 (Fig. 1). This result revealed that imazamethabenz and Pin1 have multiple combination modes.

Further examination revealed that, compared with the reference compound, compound C (13), in the crystal complex, the benzene ring of imazamethabenz did not completely occupy the binding site and had no binding group with the prolyl combination cavity, which consists of the side chains Gln131, Phe134, and His157 (Fig. 1). Hence, introducing an aromatic ring in the 1,2-position of the benzene ring would increase the number of groups that bind to the prolyl combination cavity, and this phenomenon would boost the binding force between this type of compound and Pin1. This result may help to improve imazamethabenz derivatives as selective Pin1 inhibitors.

Inhibitory activity of Pinl. The present study used a chymotrypsin coupling experiment, designated Suc-Ala-Glu-Pro-Phe-pNA as the substrate, and evaluated the inhibitory effect of the target compound on Pin1 (16). Imazamethabenz inhibited the activity of the Pin1 enzyme in vitro (Fig. 2), and its inhibitory activity $\left(\mathrm{IC}_{50}=6.67 \mu \mathrm{M}\right)$ was equivalent with various Pin1 enzyme inhibitors, as previously reported (17). Based on this result, the capacity of imazamethabenz to induce apoptosis in tumor cells was further surveyed.

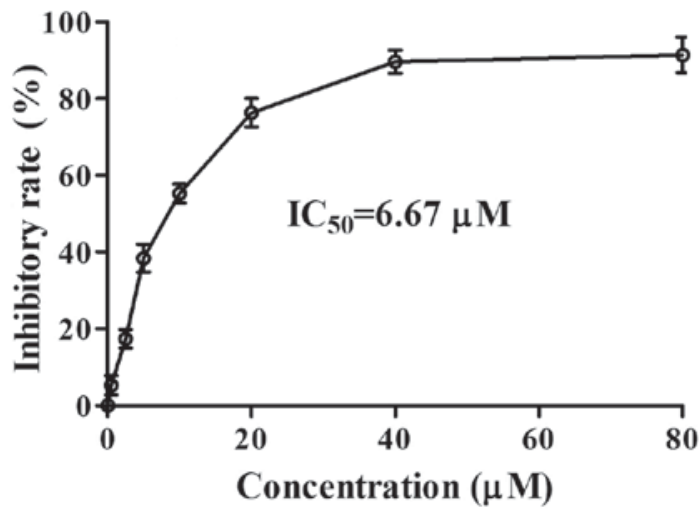

Figure 2. Inhibitory effect of imazamethabenz on Pin1.

Imazamethabenz inhibits survival, migration and invasion of $M C F-7$. Pin 1 overexpression induces cell proliferation and inhibits apoptosis through multiple mechanisms (3-5). Proteins associated with malignant tumors, including VEGF, MMP9, B cell lymphoma-2, c-myc and p53, as well as nuclear factor- $\kappa \mathrm{B}$ and cyclin $\mathrm{E}$, are active substrates of Pin1 (18-20). Hence, it was hypothesized that Pin1 inhibition in tumor cells overexpressing Pin1 would inhibit certain pathological features that are important for tumor cell survival.

The results of the MTT assay are depicted in Fig. 3A. At a concentration of 1-80 $\mu \mathrm{M}$ imazamethabenz significantly inhibited HepG-2 cell proliferation and the $\mathrm{IC}_{50}$ value was $11.32 \mu \mathrm{M}$. Western blot experiments were then performed following incubation of MCF-7 cells with imazamethabenz for 4 days. The protein expression levels of VEGF and MMP9 

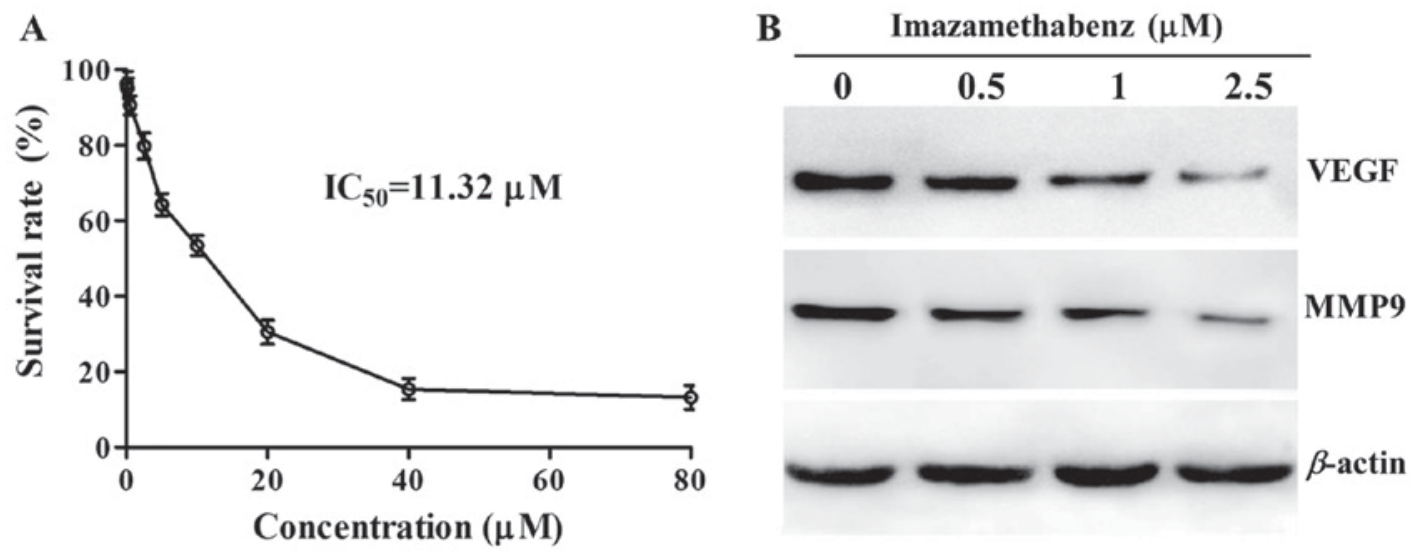

Figure 3. (A) Cytotoxicity of imazamethabenz on MCF-7 cells, measured by MTT assay. (B) VEGF and MMP9 protein expression levels following imazamethabenz treatment, as measured by western blotting. VEGF, vascular endothelial growth factor; MMP9, matrix metalloproteinase 9.
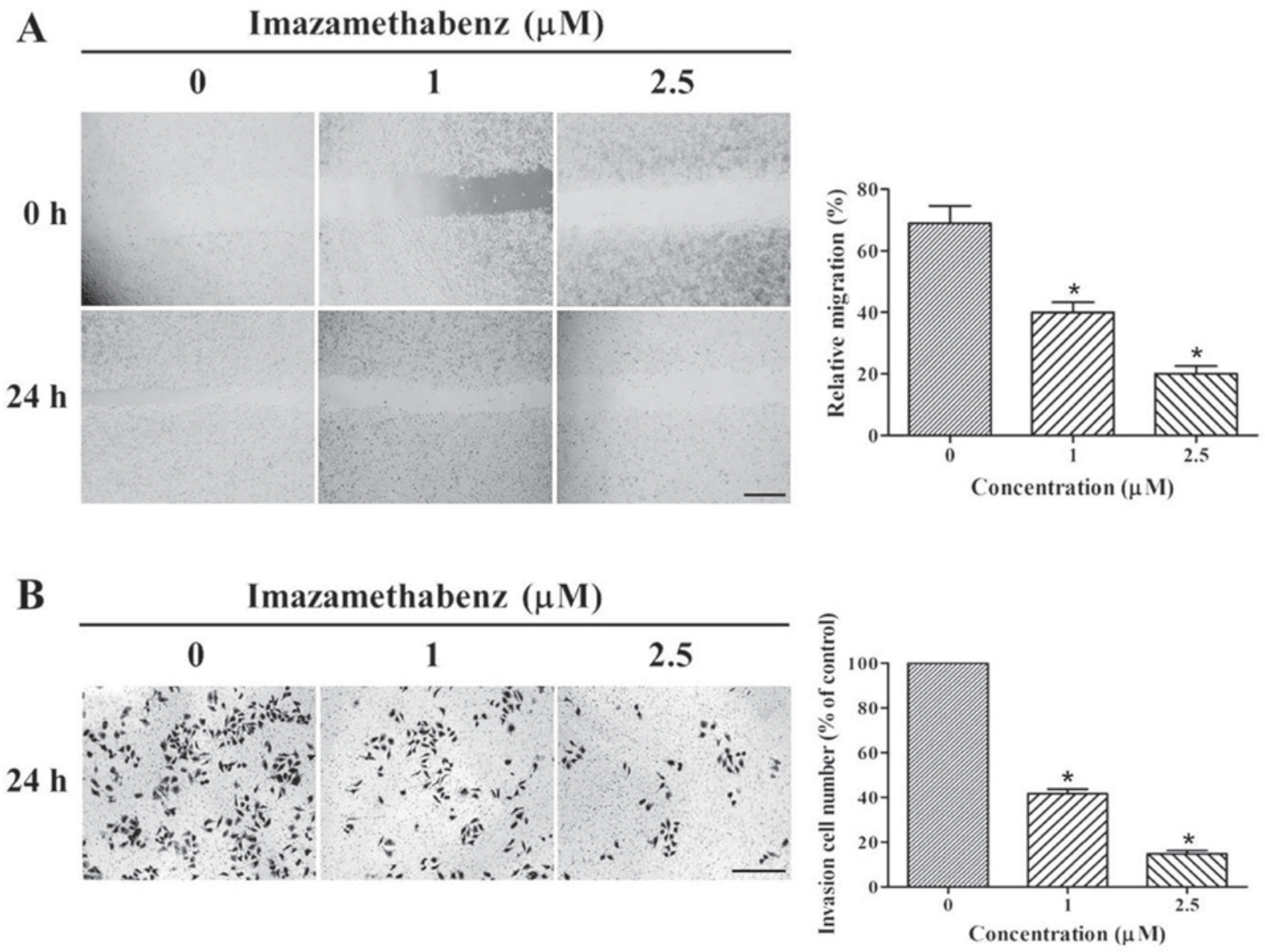

Figure 4. MCF-7 cells were treated with imazamethabenz for $24 \mathrm{~h}$, and (A) migration (scale bar, $200 \mu \mathrm{m}$ ) and (B) invasion (scale bar, $100 \mu \mathrm{m}$ ) were determined by wound healing assay and Matrigel assay, respectively. "P $<0.05$ vs. $0 \mu \mathrm{M}$ control.

were reduced in the MCF-7 cells following exposure to imazamethabenz, in a concentration dependent manner (Fig. 3B). The capacity of imazamethabenz to inhibit MCF-7 cellular migration and invasion was then assessed through further wound healing assays and transwell invasion assays, respectively. The results of the wound healing assay are depicted in Fig. 4A. Following $24 \mathrm{~h}$ incubation of imazamethabenz with MCF-7 cells, the size of the scratched region decreased less than in cells incubated without imazamethabenz. The migration rates for 1 and $2.5 \mu \mathrm{M}$ imazamethabenz were 40.84 and $19.32 \%$, respectively $(\mathrm{P}<0.05)$; therefore imazamethabenz has an inhibitory effect on endothelial cell migration. Based on the results of wound healing assay, Matrigel invasion assays were used to observe the influence of imazamethabenz on the in vitro invasion ability of MCF-7 cells, and the results are depicted in Fig. 4B. Following treatment of MCF-7 cells with 1 or $2.5 \mu \mathrm{M}$ imazamethabenz for $24 \mathrm{~h}$, the number of transmembrane cells visibly decreased in a dose-dependent 
manner, and the invasion ability of the cells was inhibited. Furthermore, compared with the control group, the number of invading cells decreased to 43.54 and $15.67 \%$, respectively $(\mathrm{P}<0.05)$. This finding indicated that Pin 1 may be an important molecule involved in the process of tumor cell migration and invasion.

To the best of our knowledge, the present study demonstrated for the first time that the imidazoline ketone herbicide, imazamethabenz, is a potential small-molecule inhibitor of Pin1. The molecular docking experiment revealed the binding mode of imazamethabenz and laid a foundation for further structural modification. A previous study performed a Pin1 enzyme inhibition experiment revealed that the enzyme inhibitory ability of imazamethabenz is able to compete with that of other Pin1 inhibitors (17). Furthermore, imazamethabenz inhibited the activity of Pin1 in vitro. Furthermore, the mechanism of imazamethabenz in inducing cellular apoptosis and a decrease in cell survival, migration and invasion may be exerted by regulating phosphorylated protein activity in the cell cycle. Under the effect of low concentrations of chemotherapeutics, which do not influence the activity of cellular survival, imazamethabenz effectively inhibited the migration and invasion of breast cancer cells and decreased the levels of VEGF and MMP9, which are involved in angiogenesis and metastasis, respectively. In conclusion, based on these results, imazamethabenz is able to inhibit tumor growth and migration by influencing protein Pin1, which is associated with cell cycle control, indicating imazamethabenz may be used as a potential compound for future tumor treatments.

\section{Acknowledgements}

The authors would like to thank the National Nature Science Foundation of China (grant no. 81302701) and the Grants for Scientific Research of BSKY (grant. no. XJ201226) from Anhui Medical University, that supported the present study.

\section{References}

1. Lu KP, Hanes SD and Hunter T: A human peptidyl-prolyl isomerase essential for regulation of mitosis. Nature 380: 544-547, 1996.

2. Bao L, Kimzey A, Sauter G, Sowadski JM, Lu KP and Wang DG: Prevalent overexpression of prolyl isomerase Pin1 in human cancers. Am J Pathol 164: 1727-1737, 2004.

3. Yeh ES and Means AR: PIN1, the cell cycle and cancer. Nat Rev Cancer 7: 381-388, 2007.
4. Sorrentino G, Comel A, Mantovani F and Del Sal G: Regulation of mitochondrial apoptosis by Pin1 in cancer and neurodegeneration. Mitochondrion 19: 88-96, 2014.

5. Lin $\mathrm{CH}$, Li HY, Lee YC, Calkins MJ, Lee KH, Yang $\mathrm{CN}$ and Lu PJ: Landscape of Pin1 in the cell cycle. Exp Biol Med (Maywood) 240: 403-408, 2015.

6. Keune WJ, Jones DR and Divecha N: PtdIns5P and Pin1 in oxidative stress signaling. Adv Biol Regul 53: 179-189, 2013.

7. Marsolier J and Weitzman JB: Pin1: A multi-talented peptidyl prolyl cis-trans isomerase and a promising therapeutic target for human cancers. Med Sci (Paris) 30: 772-778, 2014 (In French).

8. Yoon HE, Kim SA, Choi HS, Ahn MY, Yoon JH and Ahn SG: Inhibition of Plk1 and Pin1 by 5'-nitro-indirubinoxime suppresses human lung cancer cells. Cancer Lett 316: 97-104, 2012.

9. Zhu L, Jin J, Liu C, Zhang C, Sun Y, Guo Y, Fu D, Chen X and $\mathrm{Xu} \mathrm{B}$ : Synthesis and biological evaluation of novel quinazoline-derived human Pin1 inhibitors. Bioorg Med Chem 19: 2797-2807, 2011

10. Nakagawa H, Seike S, Sugimoto M, Ieda N, Kawaguchi M, Suzuki T and Miyata N: Peptidyl prolyl isomerase Pin1-inhibitory activity of D-glutamic and D-aspartic acid derivatives bearing a cyclic aliphatic amine moiety. Bioorg Med Chem Lett 25: 5619-5624, 2015.

11. Newsome WH and Collins PG: Determination of imazamethabenz in cereal grain by enzyme-linked immunosorbent assay. Bull Environ Contam Toxicol 47: 211-216, 1991.

12. Zhang Y, Daum S, Wildemann D, Zhou XZ, Verdecia MA, Bowman ME, Lücke C, Hunter T, Lu KP, Fischer G and Noel JP: Structural basis for high-affinity peptide inhibition of human Pin1. ACS Chem Biol 2: 320-328, 2007.

13. Dong L, Marakovits J, Hou X, Guo C, Greasley S, Dagostino E, Ferre R, Johnson MC, Kraynov E, Thomson J, et al: Structure-based design of novel human Pin1 inhibitors (II). Bioorg Med Chem Lett 20: 2210-2214, 2010.

14. Kofron JL, Kuzmic P, Kishore V, Colón-Bonilla E and Rich DH: Determination of kinetic constants for peptidyl prolyl cis-trans isomerases by an improved spectrophotometric assay. Biochemistry 30: 6127-6134, 1991.

15. Yuan SS, Hou MF, Hsieh YC, Huang CY, Lee YC, Chen YJ and Lo S: Role of MRE11 in cell proliferation, tumor invasion, and DNA repair in breast cancer. J Natl Cancer Inst 104: 1485-1502, 2012.

16. Liu C, Jin J, Chen L, Zhou J, Chen X, Fu D, Song H and Xu B: Synthesis and biological evaluation of novel human Pin1 inhibitors with benzophenone skeleton. Bioorg Med Chem 20: 2992-2999, 2012.

17. Min SH, Zhou XZ and Lu KP: The role of Pin1 in the development and treatment of cancer. Arch Pharm Res 39: 1609-1620, 2016.

18. Lin CH, Li HY, Lee YC, Calkins MJ, Lee KH, Yang CN and Lu PJ: Landscape of Pin1in the cell cycle. Exp Biol Med (Maywood) 240: 403-408, 2015.

19. Litchfield DW, Shilton BH, Brandl CJ and Gyenis L: Pin1: Intimate involvement with the regulatory protein kinase networks in the global phosphorylation landscape. Biochim Biophys Acta 1850: 2077-2086, 2015.

20. Kim MR, Choi HS, Heo TH, Hwang SW and Kang KW: Induction of vascular endothelial grow th factor by peptidyl-prolyl isomerase Pin 1 in breast cancer cells. Biochem Biophys Res Commun 369: 547-553, 2008. 